\title{
A CASE OF PROBABLE PARAFFIN-OIL TLMOR
}

JOHN H. STOKES, M.D.

Chief of Section on Dermatology and Syphilology, Mayo Clinic

AND

\author{
A. J. SCHOLL, JR., M.D. \\ Fellow in Urology, The Mayo Foundation \\ ROCHESTER, MINN.
}

In March, 1920, Mook and Wander ${ }^{1}$ described for American literature, on the basis of a considerable series of cases, a foreign-body tumor of the arm, essentially a modification of the familiar "paraffinoma," following the hypodermic administration of adulterated camphorated oil in which the olive oil of the pharmacopeial preparation was replaced by liquid petrolatum. The preparation apparently had been used in the treatment of influenzal pneumonia. Clinically the paraffin-oil tumor described by Mook and Wander consists of a brawny indurated and nodular infiltration of the arm having the histologic structure of a granuloma in the inflammatory phase and of a paraffinoma in the quiescent or fibrous stage.

\section{CASE REPORT}

Clinical Appearance of Tumor.-The clinical appearance of the tumor in the case reported was typical of the inflammatory phase. Chains and nodules extended from the lesion in the larger part of the anterior and posterior surfaces of the left upper arm into the axilla and from the lower margin of the lesion downward toward the elbow. This feature of the clinical picture, as Mook and Wander have pointed out, is especially likely to suggest tuberculosis or malignant neoplasia. Our clinical diagnosis of paraffinoma was subsequently confirmed by Drs. Wile, Foerster, and Irvine, who saw the patient while visiting the clinic. Mook and Wander have pointed out that tuberculosis is the usual diagnosis made by the general pathologist in these cases because the granulomatous architecture, with whorls of epithelioid cells and Langhans' giant cells, inevitably suggests such a diagnosis to one who has not seen the clinical picture.

Microscopic Examination.-In our case a large piece of tissue was excised for diagnosis. In the tissue excised in this case were innumerable "tubercles" consisting of dense whorls of epithelioid cells with giant cells in and between the whorls (Figs. 1 and 2). The Swiss-cheese structure is much less conspicuous than in the paraffinoma, owing to the greater fluidity of the oil and the consequent wide distribution of the foreign substance. Necrosis may occur in such tumors, but was not conspicuous in our case. Broders believes that there was evidence of caseation, although we were unable to identify it in the

1. Mook, W. H., and Wander, W. G.: VII. Camphor Oil Tumors, Arch. Dermat. \& Syph. 1:304-318 (March) 1920. 



Fig. 1.-Pseudotulercles with giant cells; paraffin section.

Fig. 2.-Granulomatous structure of tissue; oil vacuoles, and psendotubercles.
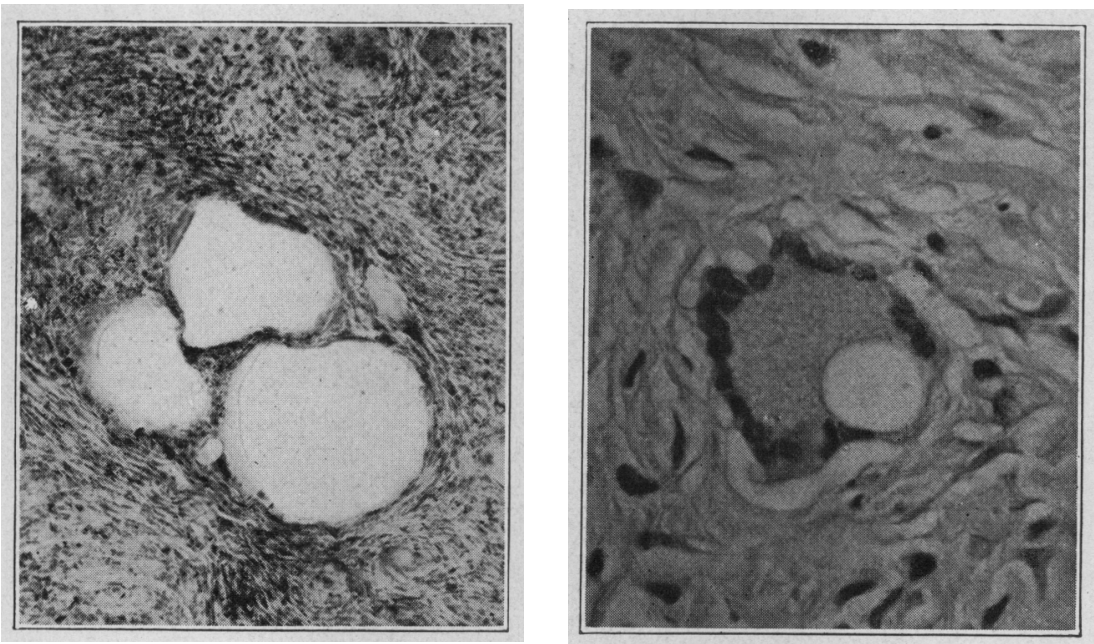

Fig. 3.- "Swiss cheese" appearance due to encapsulated paraffin oil. Frozen section stained with sudan III (oil staining light yellow) showing a film of oil still adherent to the sides of the vacuoles.

Fig. 4.-Giant cell enclosing oil vacuole. 
portion studied by us. It is worth while to recall that Cornet and Kossel" and Lewandowsky" have pointed out that the formation of tubercles without caseation may be interpreted as a foreign-body reaction to the tubercle bacillus. The high lipoid content of the organism may be compared to the paraffin oil in its power to produce a tuberculoid reaction of the type seen in this case. Vacuolation with beginning fibrous encapsulation of the oil was apparent. The efforts of the proponents of paraffinoma were centered on a demonstration of the presence of the oil in the vacuoles. This was accomplished by one of us (Scholl), who in cooperation with Dr. Robertson prepared sudan III preparations, in which the foreign oil could be distinguished from the human fat by the fact that it stains a light yellow rather than the deep orange of true fat. In a photomicrograph of such a preparation the thin layer of paraffin oil could he demonstrated still adherent to the wall of the vacuole (Fig. 3). A number of the giant cells contain what are apparently large oil vacuoles (Figs. 4 and 5 ).

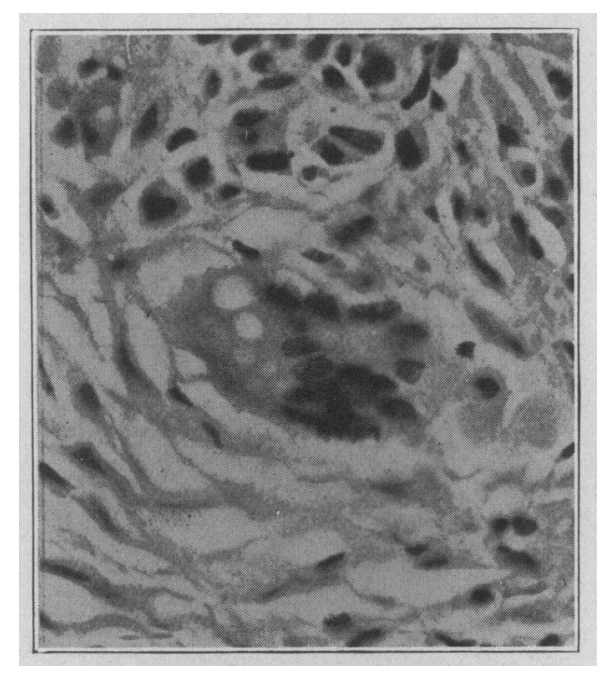

Fig. 5.-Giant cell and oil vacuoles.

Animal inoculations of paraffin oil have been made, but considerable time must elapse before a decisive result can be expected. The proponents of the diagnosis of tuberculosis thoroughly searched the excised tissue for tubercle bacilli and made two guinea-pig inoculations, both of which have been negative. No acid-fast bacilli have been demonstrable in the tissue examined.

History.-The deceptive history and peculiar sequence of events in this case deserve special mention. The patient does not recall having received anything hypodermically except typhoid vaccine in the army. Eighteen months before, however, he had had influenzal pneumonia and was unconscious from morning until evening of one day. In all probability he received the oil hypo-

2. Cornet, G., and Kossel, H.: Tuberkulose, in Kolle. W., and von Wassermann, A.: Handbuch der pathogenen Mikroorganismen, Jena, Fischer 5:391$548,1913$.

3. Lewandowsky, F.: Experimentelle Studien über Hauttuberkulose, Arch. f. Derınat. u. Syph. 98:335-398, 1909. 
dermically during this time. He had no symptoms from ine arm. A few weeks before the present lesion appeared he cut his hand on a cow's horn, and a pyogenic infection ensued with involvement of the regional lymph node (epitrochlear) and much swelling. The tumor then developed in the upper arm (Figs. 6 and 7). Mook and Wander have pointed out that the oil may produce no ill effect until a disturbance of circulatory equilibrium occurs, whereupon granulomatous and foreign-body reaction sets in. As part of the clinical evidence against tuberculosis it should be pointed out that inoculation tuberculosis in the skin always produces a tuberculous lesion at the site of inoculation

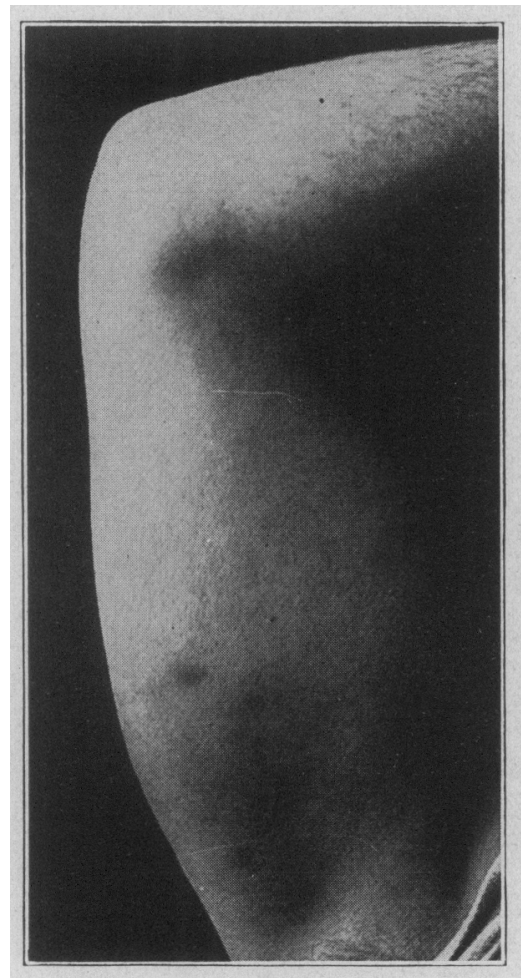

Fig. 6.--Rather unsatisfactory photograph of location of lesion. Infiltration covered surface of triceps of right arm.

in addition to the metastatic involvement, and that this metastatic involvement is of the regional lymph nodes rather than of the tissues at large. In this case there was no sign of tubercle in the scar from the trauma.

Treatment.-The treatment employed consisted of wet dressings for a period in order to favor the transition from the inflammatory into the quiescent phase. When the tumid induration and purplish discoloration had disappeared an extensive surgical excision of the affected tissue was performed. The tumid area was allowed to granulate and a skin graft was then made. The therapeutic result so far has been satisfactory. 


\section{COMMENT}

In view of the probability that a considerable amount of adulterated camphorated oil has been used during the influenza epidemics of the past two years, it would seem that as much medical publicity as possible

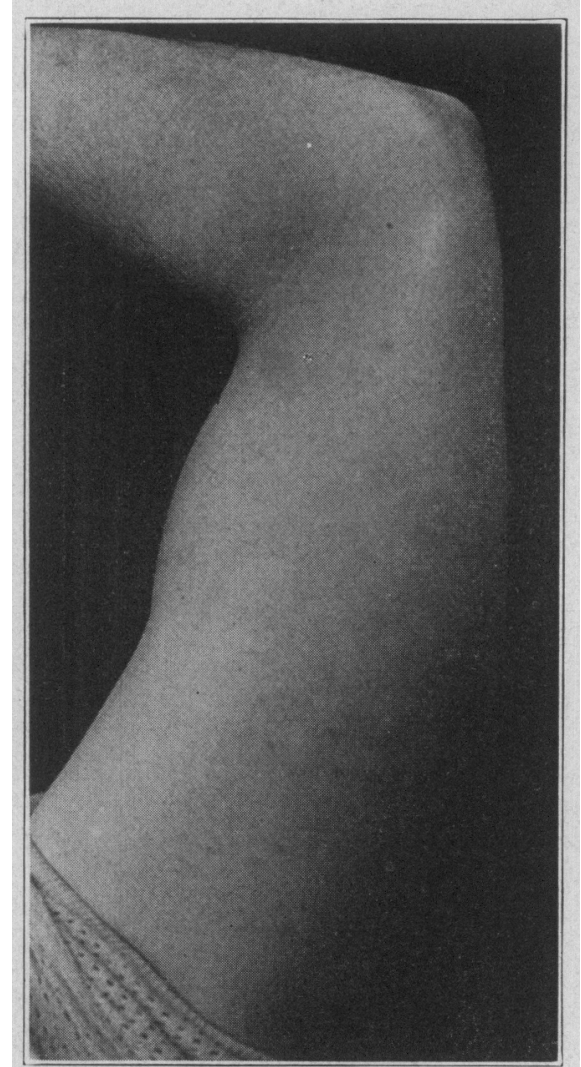

Fig. 7.-Infiltration covered surface of upper outer surface of biceps of right arm.

should be given to the camphorated-oil tumor, as its deceptive histology and misleading clinical appearance are likely to result in the diagnosis of tuberculosis and of malignant neoplasia. 